\title{
Estudiantes Coreanos de Español y Renuencia a Hablar en Clase. ¿Realidad o Mito?
}

\author{
JORGE DANIEL MENDOZA PUERTAS \\ Universidad de Ulsan (Corea del Sur)
}

Recibido: 10 diciembre 2016 / Aceptado: 1 mayo 2017

ISSN: 1698-7467

\begin{abstract}
RESUMEN: En las últimas décadas, la imagen pasiva y renuente a hablar del estudiante asiático ha sido un tema recurrente y controvertido en el área didáctica del inglés, sin embargo apenas existen estudios en el área de la didáctica del ELE, y mucho menos realizados con estudiantes coreanos. En estas páginas analizamos la renuencia a hablar en clase de los estudiantes universitarios de español en Corea empleando una metodología cuantitativa. El trabajo estadístico, basado en la Reticence Scale-12, nos permitirá conocer tanto las razones de su renuencia como si existe alguna relación entre esta y el género de los estudiantes o entre la primera y su nivel de español.
\end{abstract}

Palabras clave: ELE, renuencia a hablar, estudiantes universitarios, Corea del sur.

\section{Korean students of Spanish and communicative reticence. Myth or reality?}

ABSTRACT: In the last few decades, researchers have discussed Asian students' passiveness and reticence in English as Foreign Language classrooms. Nevertheless, there are few articles on this topic in Spanish as a Foreign Language field. In this paper, we analyze the reticence of pregraduate Korean students of SFL, applying quantitative methodology. In this regard, using the Reticence Scale 12, we conducted a survey with 200 students. The statistical data indicate not only the reasons why students are reticent but any anticipated relationship among gender, proficiency and reticence.

Keywords: SFL, communicative reticence, pregraduate students, South Korea.

\section{INTRODUCCIÓN}

Dentro de los actuales enfoques de enseñanza de lenguas extranjeras la importancia de la participación oral del alumnado en las clases está fuera de toda duda. En las últimas décadas la investigación desarrollada dentro del área de la lingüística aplicada ha venido confirmando que, más allá de la importancia del input, el output o producción posee una serie de funciones propias dentro del proceso de aprendizaje de toda lengua (Swain, 1985; Swain y Lapkin, 1995). Sin embargo, el éxito de la puesta en práctica e implementación de estos nuevos enfoques es variable según el país del que se trate y el espacio geocultural al que este pertenezca ${ }^{1}$. A este respecto, la aplicación de la metodología comunicativa en las naciones de Asia oriental ha generado importantes debates tanto en torno al perfil del

${ }^{1} \mathrm{Li}$ (1998: 698) revisa la bibliografía que alerta de estas dificultades en países como China, Japón, Vietnam, Pakistan, Singapur, Filipinas o Sudáfrica (ibíd.: 680, 681). Jeon (2009: 125) y Guilloteaux $(2004: 55,56)$ hacen referencia a la dificultad de implementación y aplicación de los enfoques comunicativos en los países asiáticos. En todos estos textos pueden observarse las dificultades de diversa índole que afectan a la aplicación del método comunicativo en Corea del Sur. 
aprendiente como en torno a la mayor o menor adecuación de estos métodos de origen occidental en contextos socioculturales y educativos tan diferentes ${ }^{2}$. Es así como el estudiante asiático ha sido tradicionalmente caracterizado por la literatura como un aprendiente pasivo, reacio a hablar en clase y, por tanto, silencioso. Especialmente en el área del inglés como lengua extranjera, la atención prestada a esta cuestión ha sido importante, sobre todo debido a la preocupación del profesorado nativo ante actitudes que resultan opuestas a aquellas requeridas por los enfoques más orientados a la comunicación. En este sentido no podemos olvidar, como exponen Arnold y Fonseca (2004: 52), que el éxito o el fracaso de estos enfoques "depende en gran parte de la participación y el aprovechamiento del alumno en actividades donde practican las destrezas orales"; de modo que si el alumno se muestra renuente a participar "se verán seriamente mermadas sus posibilidades de lograr la competencia comunicativa". El desconocimiento general, por parte de muchos profesores extranjeros, acerca de las razones que subyacen a esa actitud del estudiantado ha ocasionado malentendidos o interpretaciones poco acertadas sobre la misma. Así Donald (2010) nos dice que si los estudiantes no participan verbalmente en las tareas programadas para las clases, los profesores tenderán a pensar que estos no tienen interés en aprender. En consecuencia, en un intento de comprender mejor este fenómeno, un importante número de investigaciones ha ido encaminado a acercarse a las razones que motivan dicho comportamiento en las clases de lenguas extranjeras. A este respecto, son diversas las razones que han sido señaladas: el respeto hacia la figura del profesor, la preservación de la imagen pública, el mantenimiento de la armonía, la modestia, la importancia del silencio frente al lenguaje, la escasa competencia en la lengua meta, el miedo a cometer errores, la falta de confianza, la ansiedad, la timidez, las consecuencias de una educación centrada en la figura del profesor (Bao, 2014; Chang, 2011; Cheng, 2000; Cortazzi y Jin, 1996; Donald, 2010; He, 2008; Hsieh, 2015; Li y Jia, 2006; Liu, 2005a, 2005b; Liu y Jackson, 2008; Liu y Littlewood, 1997; Liu, Zhang y Lu, 2011; Shimizu, 2006; Soo y Goh, 2013; Tran, 2013; Tsui, 1996) así como otras causas menos reiteradas. Las investigaciones centradas en el alumnado coreano comparten buena parte de las razones anteriores, incluyendo, entre ellas, alguna que otra causa proveniente del sustrato cultural de Corea, por ejemplo, el chemyon (Bao, 2014; Chen, 2003; Choe, 1995; Choi, 2015; Kim, 2013; Lee, 2009).

Por otro lado, no faltan investigadores que rechazan este perfil poco activo del alumno asiático y, por ende, esa actitud renuente a participar en clase. Entre ellos, Kumaravadivelu (2003) considera que esa imagen responde a un estereotipo creado por los profesores extranjeros para poder lidiar con lo desconocido haciéndolo más manejable. Por otro lado, según Cheng (2000), estos alumnos no se encuentran predispuestos culturalmente a ser reacios a hablar en clase y, al igual que Littlewood $(1999,2000)$, lamenta la generalización que sobre este tema se ha producido en la literatura. En esta misma línea, Tran (2013) desmitifica la imagen estereotipada de aprendices pasivos con la que han sido etiquetados alumnos de muy diversos países del continente asiático.

\footnotetext{
${ }^{2}$ Véase García Santa-Cecilia (2009).
} 


\section{El constructo de la renuencia}

El primero en introducir el constructo de la renuencia dentro del campo de la comunicación oral fue Phillips en 1965, al destacar que ciertas personas presentaban dificultad para comunicarse en toda una serie de situaciones. En su definición original Phillips (1965) describía la renuencia a hablar como un trastorno de ansiedad basado en la personalidad. Esta idea será refinada con el tiempo, de manera que a partir de 1977 pasará a definirla como un problema de inadecuadas habilidades comunicativas (Phillips, 1977, 1984, 1986, 1991). De acuerdo con este autor, la persona renuente evita la comunicación bien debido a su falta de habilidad para la interacción social y para la actuación en público, o bien debido a que se cree carente de estas habilidades. De este modo, la idea central del concepto de renuencia se resume en la siguiente afirmación: cuando las personas evitan la comunicación es porque piensan que perderán más hablando que permaneciendo callado (Phillips, 1984: 52).

La conceptualización de Phillips incluye dos dimensiones dentro del fenómeno de la renuencia comunicativa, una cognitiva y otra conductual. El componente cognitivo se relaciona con la adherencia del sujeto a un conjunto de creencias ${ }^{3}$ o "mitos" que condiciona su propia percepción como hablante y lo lleva a evitar la comunicación (Keaten, Kelly y Finch, 2000). Según Phillips, estas creencias contribuyen a crear el ciclo de la renuencia al generar una conducta con la que se evita la interacción en un intento de no parecer ridículo y de no caer en la manipulación comunicativa. Si bien, al mismo tiempo, este hecho provoca que las personas afectadas no desarrollen adecuadamente sus habilidades para comunicarse y que, conocedoras de su ineptitud, se encierren en su propio silencio. Keaten, Kelly y Finch (2000) corroboraron que aquellos estudiantes que podían definirse como renuentes a hablar se mostraban de acuerdo con algunas de las creencias señaladas por Phillips en su constructo, pero no con todas.

La conceptualización de la renuencia a la comunicación oral iría evolucionando durante más de tres décadas. Los datos recabados por distintos investigadores, así como los resultados de sus propias investigaciones llevaron a Keaten y Kelly (2000) a presentar una modificación del constructo que Phillips había ido perfilando. En este sentido, Keaten y Kelly añaden una nueva dimensión al mismo: "The theoretical model of reticence we present maintains the behavioral and cognitive dimensions identified by Phillips, albeit with some modifications, but adds an affective component" (2000: 168). Estos autores parten de una definición del concepto de renuencia que varía respecto a la de Phillips: "When people avoid communication because they believe it is better to remain silent than to risk appearing foolish, we refer to this behavior as reticence" (íd.)

Esta definición de la renuencia a hablar se basa en un modelo económico de la interacción: el individuo decide comunicarse o no, según su percepción de aquello que puede lograr o de aquello que puede perder al establecer dicha interacción. En este sentido, los sujetos renuentes tienden a subestimar las recompensas y a sobreestimar las sanciones que pueden recibir como consecuencia de su actuación comunicativa. Así, estos suelen experimentar un gran miedo a la evaluación negativa y presentar una gran sensibilidad frente a la opinión de los demás, por lo que sufren grandes niveles de ansiedad (íd.).

\footnotetext{
${ }^{3}$ Para una aproximación a las creencias señaladas por Phillips, véase Keaten y Kelly (2000).
} 
Hasta 1997 no aparecería un método válido para medir este fenómeno, ya que fue en ese año cuando Keaten, Kelly y Finch publicaron una escala destinada a medirlo ${ }^{4}$. Esta guardaba relación con una de las últimas conceptualizaciones de Phillips, en la que se afirmaba que la carencia de habilidades que definía el constructo estaba vinculada a los cánones de la retórica: inventio, dispositio, elocutio, memoria y actio. La Reticence Scale-24 (RS-24) se encontraba compuesta por las siguientes dimensiones: ansiedad, conocimiento (inventio), tiempo, organización de las ideas (dispositio), forma de hablar y memoria. Cada dimensión estaba compuesta por 4 ítems, y cada uno de los cuales debía ser respondido usando una escala Likert (Keaten, Kelly y Finch, 1997). La finalidad de estas 24 afirmaciones era conocer cómo los sujetos aplicaban sus emociones y habilidades relacionadas con la comunicación en situaciones sociales. Las puntuaciones más bajas obtenidas en la escala indicarían niveles menores de renuencia frente a aquellas puntuaciones más elevadas que señalarían un nivel de renuencia mayor (íd.).

\section{OвJetivos}

En este trabajo pretendemos acercarnos al problema de la renuencia a participar oralmente en el contexto de las clases universitarias de ELE en Corea. Seguimos, así, la línea iniciada por otras investigaciones llevadas a cabo en diversos países de Asia dentro el ámbito del inglés (Chalak y Baktash, 2015, 2016; Delima, 2012; Musytarif, 2015; Soo y Goh, 2013). Este acercamiento pretende responder, desde un enfoque cuantitativo, a las siguientes cuestiones:

a) ¿Cuántos estudiantes encuestados presentan un grado relevante de renuencia a hablar?

b) ¿Cuáles son las principales causas señaladas por nuestros estudiantes?

c) ¿Existe una relación entre género y renuencia?

d) ¿Existe una relación entre el nivel de español y la renuencia a hablar?

\section{Procedimiento}

La presente investigación ha sido realizada en Corea del Sur, concretamente en la Universidad de Ulsan y en la Universidad Católica de Daegu. Ambas son universidades privadas que cuentan entre su catálogo de titulaciones con grados destinados tanto al aprendizaje de la lengua española como al conocimiento de los ámbitos político, social, literario, etc. de España y los países latinoamericanos; y que poseen, por tanto, departamentos relacionados con el español y las demás áreas asociadas.

El instrumento utilizado para este estudio fue la Reticence Scale-12 (RS-12), destinada a medir el grado de renuencia a hablar de nuestros participantes. Esta escala supone una

\footnotetext{
${ }^{4}$ Si bien Phillips y Erickson, en 1965, crearon una escala para medir la renuencia, esta era demasiado extensa (85 ítems) y resultaba poco viable para someterla a análisis estadísticos (Keaten, Kelly y Finch, 1997). Phillips terminaría abandonándola y prefiriendo las entrevistas para identificar a los sujetos con problemas.
} 
versión reducida de la anteriormente expuesta RS-24. La RS-12 mide las seis dimensiones de la renuencia ya citadas: sensaciones de ansiedad, conocimiento del tema de conversación, habilidades de organización del tiempo, organización de las ideas, forma de hablar y memoria; si bien los ítems para cada una de estas dimensiones quedan reducidos a dos ${ }^{5}$ (Kelly, Keaten, Hazel y Williams, 2010). Para medir cada ítem se empleó una escala Likert de 5 puntos, que oscilaba desde el 1 (totalmente en desacuerdo) al 5 (totalmente de acuerdo). Respecto a la fiabilidad del cuestionario, si bien investigaciones previas ya demostraron que la RS12 resulta un instrumento adecuado para medir el fenómeno que nos ocupa (Chalak y Baktash, 2015; Kelly, Keaten, Hazel y Williams, 2010; Soo y Goh, 2013), en nuestro análisis de su consistencia interna obtuvimos un alfa de Cronbach de 0,907 . Un valor que otorga una muy buena fiabilidad a la medición del constructo en la muestra concreta utilizada.

La encuesta fue distribuida entre el primer y el segundo semestre del año 2016 entre un total de 200 estudiantes pertenecientes a la carrera de español de las universidades citadas. Entre ellos, 130 encuestados eran alumnos de la Universidad de Ulsan y 70 de la Universidad Católica de Daegu. Los participantes fueron seleccionados entre los alumnos pertenecientes a los cursos de $2 .^{\circ}, 3 .^{\circ}$ y $4 .^{\circ}$ de grado, habiéndose descartado a los estudiantes de primer año debido a sus importantes limitaciones en la lengua meta. Más concretamente, participaron 99 estudiantes de $2 .^{\circ}$ curso, 50 de $3 .^{\circ}$ y 51 de $4 .^{\circ}$ curso. La distribución por sexos fue de 81 hombres y 119 mujeres. El rango de edad de los encuestados osciló entre $\operatorname{los} 19$ y $\operatorname{los} 26$ años.

El cuestionario original fue traducido del inglés a la lengua materna de los participantes, es decir, al coreano. Para la cumplimentación del mismo se les ofreció 15 minutos y el investigador siempre estuvo presente mientras se realizaba. A los encuestados se les explicó la finalidad académica de dicho cuestionario y se les garantizó su anonimato. Los datos recopilados se sometieron a análisis estadísticos utilizando para ello el paquete SPSS 22.

\section{Resultados}

En relación con la primera cuestión planteada en esta investigación, teniendo en cuenta que la puntuación máxima que puede obtenerse en el cuestionario es de $60 \mathrm{y}$ que su punto intermedio es 30, cabe destacar que Chalak y Baktash (2015) consideran altamente renuentes a los encuestados que obtuvieron una puntuación por encima de 31, mientras que O'Connell (2010) y Soo y Goh (2013) hablan de importante renuencia con un puntaje por encima de 29. En nuestro caso, hemos considerado renuentes a hablar en clase a aquellos estudiantes que obtuvieron una puntuación superior o igual a 30, mientras que estimamos poco renuentes a aquellos cuyo puntaje fue inferior a la cifra mencionada. Los resultados de nuestro estudio ofrecen una media de 35,08, una mediana de 36,00 y una moda de 38 , lo que apunta hacia un conjunto de valores que parece situarse mayoritariamente por encima de los 30 puntos. Así lo demuestra también el número de estudiantes que puede ser catalogado como reacio

\footnotetext{
${ }^{5}$ Cabe destacar que la Reticence Scale es una herramienta basada en las "creencias" o en la "percepción" del estudiante acerca de sus habilidades comunicativas en público. No toma, de este modo, en consideración la actuación del aprendiz en el aula ni la actuación del docente. No obstante, como señalan Soo y Goh (2013), se trata de un instrumento fundamental para identificar a aquellos estudiantes con tendencia a ser renuentes a comunicarse.
} 
a hablar, un total de $137(68,5 \%)$ frente a 63 de ellos $(31,5 \%)$ que mostraron una baja renuencia comunicativa. Como puede observarse, existe una mayoría muestral para la que hablar en la clase de ELE supone un problema o al menos una dificultad añadida al propio proceso de aprendizaje. Estos resultados se posicionan en la línea de aquellos ofrecidos por estudios similares realizados en otros países asiáticos (Chalak y Baktash, 2015; Musytarif, 2015; Soo y Goh 2013), donde una considerable mayoría de alumnos se mostraba reacia a participar oralmente en las aulas de inglés.

Como expusimos más arriba la Reticence Scale sirve para medir la renuencia a comunicarse oralmente en relación con seis dimensiones: ansiedad, conocimiento, tiempo, organización, forma de hablar y memoria. Una vez conocido que el número de estudiantes renuentes supera con creces a aquellos que no pueden ser calificados como tales, nos interesa saber en qué aspectos medidos por el cuestionario se apoya su renuencia. La siguiente tabla recoge tanto la frecuencia como el porcentaje de encuestados que se muestra "totalmente de acuerdo/de acuerdo", "indeciso" o "totalmente en desacuerdo/en desacuerdo" con cada una de las afirmaciones ${ }^{7}$ agrupadas por dimensiones. Es evidente que un nivel importante de renuencia vendrá marcado por un mayor acuerdo del encuestado con las afirmaciones que contiene el cuestionario:

Tabla 1: Respuestas de los encuestados a las afirmaciones de la encuesta

\begin{tabular}{|c|c|c|c|c|c|c|c|}
\hline & \multirow[t]{2}{*}{ Dimensiones y afirmaciones } & \multicolumn{2}{|c|}{$\begin{array}{l}\text { Totalmente de } \\
\text { acuerdo / De } \\
\text { acuerdo }\end{array}$} & \multicolumn{2}{|c|}{ Indeciso } & \multicolumn{2}{|c|}{ 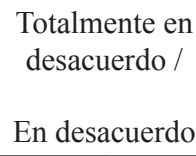 } \\
\hline & & $\mathrm{f}$ & $\%$ & f & $\%$ & $\mathrm{f}$ & $\%$ \\
\hline \multicolumn{8}{|c|}{ 1. Ansiedad } \\
\hline a) & Estoy nervioso. & 98 & 49 & 17 & 8,5 & 85 & 42,5 \\
\hline b) & Me siento tenso. & 103 & 51,5 & 15 & 7,5 & 82 & 41 \\
\hline \multicolumn{8}{|c|}{ 2. Forma de hablar } \\
\hline a) & Confundo las palabras. & 139 & 69,5 & 28 & 14 & 33 & 16,5 \\
\hline b) & Me trabo. & 128 & 64 & 34 & 17 & 38 & 19 \\
\hline \multicolumn{8}{|c|}{ 3. Memoria } \\
\hline a) & Me olvido de lo que quiero decir. & 41 & 20,5 & 35 & 17,5 & 124 & 62 \\
\hline b) & Pierdo de vista lo que quiero decir. & 22 & 11 & 33 & 16,5 & 145 & 72,5 \\
\hline \multicolumn{8}{|c|}{ 4. Organización } \\
\hline a) & Mis ideas están desordenadas. & 109 & 54,5 & 22 & 11 & 69 & 34,5 \\
\hline b) & Mis ideas están mezcladas. & 97 & 48,5 & 23 & 11,5 & 80 & 40 \\
\hline
\end{tabular}

${ }^{6}$ Por cuestiones de espacio hemos agrupado los ítems de la escala Likert que guardan relación entre sí.

${ }^{7}$ Obviamente, las afirmaciones del cuestionario se refieren al momento en el que el alumno habla español en clase. 


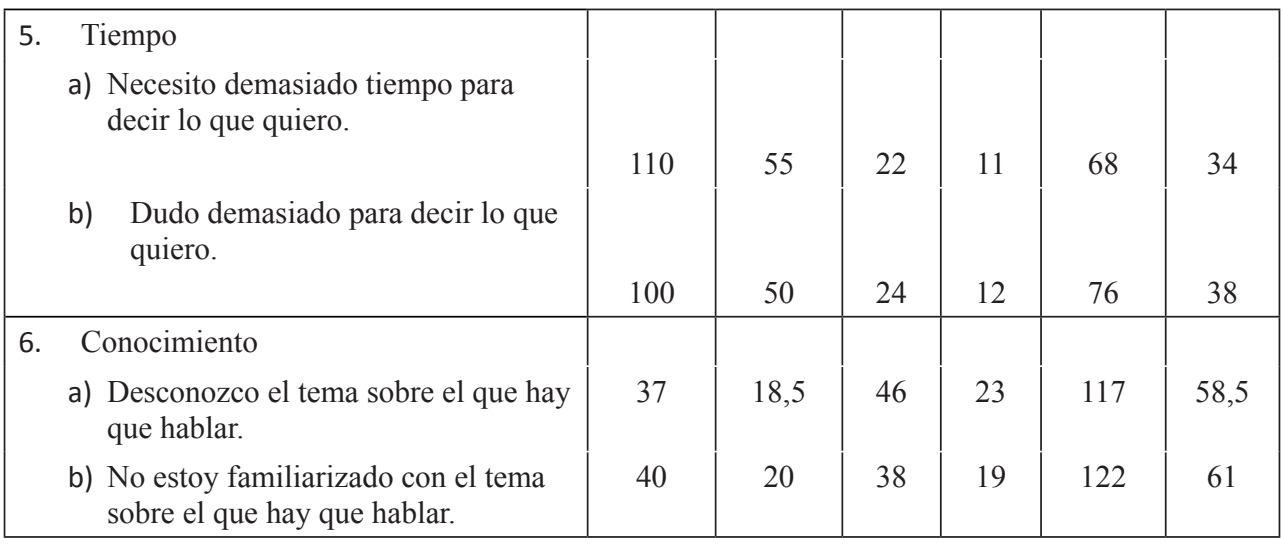

De acuerdo con la tabla previa, si nos fijamos en las respuestas indicadoras de renuencia, podemos observar tres dimensiones que destacan por encima de las demás. La primera de ellas, con una notable diferencia respecto a las otras, es la dimensión "forma de hablar", en la que el $69,5 \%$ de los encuestados considera que confunde las palabras al hablar y el $64 \%$ estima que se traba al hablar español. En segundo lugar se sitúa la dimensión "tiempo", donde el $55 \%$ de los estudiantes piensa que necesita demasiado tiempo para decir lo que quiere y el $50 \%$ estima que duda demasiado para decir lo que desea. En tercer lugar se posiciona la dimensión "organización", en la que el 54,5\% percibe que cuando habla sus ideas están desordenadas y un 48,5\% considera que las ideas aparecen mezcladas en su discurso. Por último puede apreciarse otra dimensión, si bien no tan destacada, donde también el número de encuestados que se muestra de acuerdo con sus afirmaciones es mayor que aquel que se muestra en desacuerdo; nos referimos a la dimensión "ansiedad". En ella, el $49 \%$ estima que está nervioso cuando habla español en clase y el $51,5 \%$ considera que se siente tenso cuando habla.

Otra cuestión que ha sido abordada por distintos investigadores es la relación entre género y renuencia a hablar. En este sentido, Caspi, Chajut y Saporta (2008) observaron que las mujeres suelen participar con menos frecuencia en las clases que los hombres, quienes normalmente se muestran más activos, confiados y proclives a hablar. En oposición a estos resultados, Soo y Goh (2013) no encontraron en su estudio diferencias señalables en la participación de hombres y mujeres. Por nuestra parte, los datos obtenidos en el presente trabajo apoyan los resultados de Caspi, Chajut y Saporta (2008), ya que muestran una relación significativa entre la diferencia de género y el nivel de renuencia de los encuestados. La siguiente tabla ofrece una comparación entre grados de renuencia y el género de los participantes: 
Tabla 2: Tabla de contingencia Género * Nivel de renuencia

\begin{tabular}{|c|c|c|c|c|c|}
\hline & & & \multicolumn{2}{|c|}{ Renuencia } & \multirow[b]{2}{*}{ Total } \\
\hline & & & $<30$ & $\geq 30$ & \\
\hline \multirow[t]{8}{*}{ Género } & \multirow[t]{4}{*}{ Masculino } & Recuento & 32 & 49 & 81 \\
\hline & & Recuento esperado & 25,5 & 55,5 & 81,0 \\
\hline & & $\%$ dentro del género & $39,5 \%$ & $60,5 \%$ & $100 \%$ \\
\hline & & $\%$ del total & $16 \%$ & $24,5 \%$ & $40,5 \%$ \\
\hline & \multirow[t]{4}{*}{ Femenino } & Recuento & 31 & 88 & 119 \\
\hline & & Recuento esperado & 37,5 & 81,5 & 119,0 \\
\hline & & $\%$ dentro del género & $26,1 \%$ & $73,9 \%$ & $100 \%$ \\
\hline & & $\%$ del total & $15,5 \%$ & $44 \%$ & $59,5 \%$ \\
\hline \multirow{3}{*}{\multicolumn{2}{|c|}{ Total }} & Recuento & 63 & 137 & 200 \\
\hline & & Recuento esperado & 63,0 & 137,0 & 200,0 \\
\hline & & $\%$ del total & $31,5 \%$ & $68,5 \%$ & $100 \%$ \\
\hline
\end{tabular}

Tal como puede verse en la tabla, existe una cierta diferencia entre el número de estudiantes varones que se muestra poco participativo en clase y el número de mujeres. Debido al diferente número de alumnos y alumnas que colaboraron en la encuesta, si nos centramos en los porcentajes obtenidos dentro de cada grupo, vemos que un $60,5 \%$ de los hombres pueden ser considerados renuentes a hablar frente al 73,9\% de las mujeres. Sin embargo, es la prueba de chi-cuadrado de Pearson la que corrobora lo expuesto con anterioridad. Debido a que p-valor es inferior a 0,05 (la significación asintótica es de 0,044$)^{8}$ descartamos la hipótesis nula basada en la independencia de las variables y consideramos que en nuestra muestra existe una relación entre el género y el nivel de renuencia. Es decir, la renuencia no se muestra por igual entre hombres y mujeres sino que las participantes de nuestro estudio tienden a ser más reacias a hablar que los estudiantes encuestados.

Por otro lado, atendiendo a la última pregunta planteada entre los objetivos de este trabajo, cabe destacar que, según diversos investigadores, el nivel de competencia en la lengua meta incide en la actitud más o menos participativa del estudiante en la clase de idiomas. Así, Liu y Jackson (2009), en un estudio llevado a cabo entre alumnos universitarios chinos, llegaron a la conclusión de que aquellos aprendientes cuyo dominio de la lengua era más alto mostraban una mayor inclinación a hablar en clase y una actitud más positiva hacia el uso de la lengua oral. En esta línea, Choi (2015) expone que los estudiantes coreanos

\footnotetext{
${ }^{8}$ No hay casillas que presenten un recuento menor que 5. El recuento mínimo esperado es de 25,52.
} 
de posgrado que participaron en su investigación atribuían su silencio, entre otras razones, a su escasa competencia oral dentro del área de especialidad que estudiaban. Ahora bien, la relación entre la resistencia a hablar y la falta de competencia ya había sido apuntada anteriormente en trabajos como el de Tsui (1996), Chau (1999) o Liu (2005b), entre otros.

En el entorno educativo aquí analizado, esta diferencia en el nivel de lengua es especialmente apreciable entre dos grupos de alumnos: aquellos que estuvieron estudiando español fuera de Corea en un país donde la lengua meta es el vehículo de comunicación, y aquellos otros que únicamente han estudiado español dentro del sistema educativo coreano y no han disfrutado de la anterior experiencia ${ }^{9}$. Evidentemente, los primeros gozan de una mayor competencia en la lengua frente al más limitado dominio de los segundos. En consecuencia, para medir la relación entre la renuencia a hablar de los estudiantes encuestados y su competencia en español hemos distinguido como variables la experiencia de estudio en el extrajero o la ausencia de esta. En la siguiente tabla puede apreciarse la comparación establecida entre el nivel de competencia en español y el nivel de renuencia:

Tabla 3: Tabla de contingencia Nivel de español * Nivel de renuencia

\begin{tabular}{|c|c|c|c|c|c|}
\hline & & & \multicolumn{2}{|c|}{ Renuencia } & \multirow{2}{*}{ Total } \\
\hline & & & $<30$ & $\geq 30$ & \\
\hline \multirow{8}{*}{$\begin{array}{l}\text { Nivel de } \\
\text { español }\end{array}$} & \multirow{4}{*}{$\begin{array}{l}\text { Experiencia en } \\
\text { el extranjero }\end{array}$} & Recuento & 38 & 25 & 63 \\
\hline & & Recuento esperado & 19,8 & 43,2 & 63,0 \\
\hline & & $\%$ dentro de la categoría & $60,3 \%$ & $39,7 \%$ & $100 \%$ \\
\hline & & $\%$ del total & $19 \%$ & $12,5 \%$ & $31,5 \%$ \\
\hline & \multirow{4}{*}{$\begin{array}{l}\text { Sin experiencia } \\
\text { en el extranjero }\end{array}$} & Recuento & 25 & 112 & 137 \\
\hline & & Recuento esperado & 43,2 & 93,8 & 137,0 \\
\hline & & $\%$ dentro de la categoría & $18,2 \%$ & $81,8 \%$ & $100 \%$ \\
\hline & & $\%$ del total & $12,5 \%$ & $56 \%$ & $68,5 \%$ \\
\hline \multirow{2}{*}{\multicolumn{2}{|c|}{ Recuento esperado }} & Recuento & 63 & 137 & \multirow[t]{3}{*}{200} \\
\hline & & 63,0 & 137,0 & 200,0 & \\
\hline \multicolumn{2}{|c|}{$\%$ del total } & $31,5 \%$ & $68,5 \%$ & $100 \%$ & \\
\hline
\end{tabular}

Atendiendo a los porcentajes dentro de cada categoría, puede advertirse que solo el $39,7 \%$ de los estudiantes con mayor competencia en la lengua pueden ser considerados renuentes a hablar frente al $81,8 \%$ de aquellos cuyo nivel de competencia es inferior. De

\footnotetext{
${ }^{9}$ A mediados del segundo curso los estudiantes pueden elegir estudiar español en España o en distintos países de Latinoamérica. Generalmente estas estancias oscilan entre los seis meses y un año, y suelen realizarse en centros de idiomas o programas universitarios para extranjeros.
} 
acuerdo con estos datos, la distancia entre ambos grupos es notable. A este respecto, la prueba de chi-cuadrado, con un p-valor similar a cero $^{10}$, deja constancia de la dependencia de las variables dentro de la muestra y de la relación existente entre el nivel de competencia y la resistencia a hablar en la clase de ELE.

\section{DISCUSIÓN}

A la vista de los datos previos, la muestra seleccionada se ajusta, en su mayoría, al perfil del estudiante asiático reacio a participar en clase. Las causas señaladas por los encuestados resultan ciertamente interesantes, no en vano, las principales razones quedan vinculadas a las dimensiones "forma de hablar", "tiempo" y "organización", mientras que la dimensión "ansiedad" se posiciona por detrás de estas. Los resultados difieren, por tanto, de los hallazgos de otros investigadores como Soo y Goh (2013) o Chalak y Baktash (2015, 2016), en cuyos trabajos las causas asociadas a los factores afectivos predominaban sobre las demás. De igual manera, los niveles de ansiedad reflejados en nuestra encuesta no parecen tan altos como aquellos defendidos por el constructo de la renuencia. A este respecto, es posible que la metodología comunicativa, el ambiente distendido y la perspectiva de aceptación del error como parte del aprendizaje que los actuales profesores de español aplicamos en nuestras aulas estén dando sus frutos. Puesto que la encuesta ha sido distribuida entre alumnos que ya superaron el primer curso, debemos tener en cuenta que estos han disfrutado de un proceso de adaptación al profesorado nativo y a la dinámica seguida en el aula, algo que podría actuar como un reductor de la ansiedad. En este sentido, Chalak y Baktash (2015) ya observaron que el método de enseñanza y el trato del profesor en clase influyen sobre la resistencia a hablar, de modo que esta solía ser más alta al principio del semestre e ir decreciendo a partir de mitad del mismo. Una realidad que puede haber influido en los resultados de la presente investigación.

La vinculación entre género y renuencia, destacada por autores como Caspi, Chajut y Saporta (2008), se confirma también en las páginas previas. A diferencia de la independencia entre género y renuencia hallada por Soo y Goh (2013), en la muestra aquí analizada los encuestados presentan un menor rechazo a hablar en clase frente al mayor rechazo de las encuestadas. La relación significativa corroborada entre estas dos variables parece confirmar la imagen de los estudiantes varones como menos renuentes y más inclinados a comunicarse frente aquella más inhibida y menos proclive a hablar de las mujeres.

Respecto a la relación existente entre el nivel de competencia en la lengua y el grado de resistencia a hablar, el presente estudio contradice los resultados obtenidos por Soo y Goh (2013) entre los estudiantes malayos y se posiciona en la línea de otras muchas investigaciones que ya destacaron una relacion inversa entre nivel de competencia y nivel de rechazo a comunicarse oralmente. En este sentido, las afirmaciones que obtuvieron un mayor puntaje en la escala, como "confudir las palabras" o "necesitar más tiempo para decir lo

\footnotetext{
${ }^{10}$ La significación asintótica es de 0,000 . No hay casillas que presenten un recuento menor que 5 . El recuento mínimo esperado es de 19,85.
} 
que se desea”, guardan cierta relación con el nivel de competencia oral y dominio hablado de la lengua meta.

No cabe duda, tal como han venido mostrando muy diversos estudios, de que el fenómeno de la resistencia a hablar es un hecho complejo y en el que influyen muy diversos factores. Su presencia en las aulas del continente asiático ha quedado más que constatada. Es por ello por lo que la labor del profesorado nativo, cuyos modelos de enseñanza suelen diferir bastante de los modelos tradicionales y locales, deviene esencial para contrarrestar una realidad poco deseable en las aulas de lenguas extranjeras. En primer lugar, la creación de un clima agradable que atienda a los factores afectivos y disminuya la ansiedad constituye un elemento clave contra la renuencia (Donald, 2010; Liu, 2005a; Tsui, 1996). El aumento de la confianza dentro del aula incidirá, sin duda, de manera positiva en áreas como la "forma de hablar" y la "organización", ya que llevará al estudiante a albergar menos dudas sobre su capacidad y a sentirse más decidido a usar la lengua. Por otro lado, son numerosos los investigadores que se han hecho eco de las demandas del alumnado asiático respecto a la falta de tiempo para organizar y articular su discurso en clase (Bao, 2014; Chang, 2011; Donald, 2010; Li \& Jia, 2006; Liu \& Littlewood, 1997; Tsui, 1996). Es evidente que en este punto, el profesor debe otorgar mayor tiempo al estudiante para responder sus preguntas o exponer aquello que desee, o incluso en aquellos casos en los que el alumno necesite un lapso de tiempo mayor, concederle un tiempo extra para que medite su respuesta mientras permite participar a otros aprendientes.

\section{CONCLUSIONES}

En las páginas anteriores hemos medido el fenómeno de la renuencia comunicativa en un conjunto de estudiantes de español dentro de sistema universitario coreano. La muestra elegida presenta, en su mayoría, una tendencia a ser renuente a hablar en las clases de ELE. Las principales razones sobre las que se apoya este fenómeno quedan asociadas a las dimensiones "forma de hablar", "tiempo" y "organización"; si bien nuestros alumnos no presentan altos índices de ansiedad dentro del constructo.

El presente estudio corrobora una vinculación entre género y renuencia, donde las mujeres muestran una actitud más inhibida frente a aquella menos reacia a hablar de los varones. Por otro lado, se ha comprobado que cuanto mayor es la competencia comunicativa del encuestado su grado de renuencia es menor.

La imposibilidad de contrastar los resultados de este trabajo con otros similares realizados en el ámbito de la enseñanza del español en Corea del Sur constata la necesidad de que aparezcan nuevos estudios, tanto cuantitativos como cualitativos, en torno a este tema. No en vano estas investigaciones servirían para abrir camino a la intervención docente en aras de ir ofreciendo soluciones a este complejo fenómeno, de acuerdo con el perfil del estudiante presente en el aula. 


\section{REFERENCIAS}

Arnold, J. y Fonseca, M. C. (2004). "Reflexiones sobre aspectos del desarrollo de la competencia comunicativa oral en el aula de español como segunda lengua", en F. L. Berguillos y S. Ruhstaller (cords.) (2004): La competencia lingüistica y comunicativa en el aprendizaje de español como lengua extranjera. Sevilla: Edinumen, 45-60.

Bao, D. (2014). Understanding Silence and Reticence. Ways of Participating in Second Language Acquisition. London-New York: Bloomsbury.

Caspi, A.; Chajut, E. y Saporta, K. (2008). "Participation in class and in online discussions: Gender differences", en Computers \& Education, 50: 718-724.

Chalak, A. y Baktash, F. (2015). "An Investigation on Students' Reticence in Iranian University EFL Classrooms", en International Scholarly and Scientific Research \& Innovation, 9, 8: 2554-2557.

Chalak, A. y Baktash, F. (2016). "The Relationship between Reticence and Personality Types in Iranian University EFL Classrooms", en Theory and Practice in Language Studies, 6, 5: 1000-1005.

Chang, F. (2011). "The Causes of Learners' Reticence and Passivity in English Classrooms in Taiwan", en The Journal of Asia TELF, 8, 1: 1-22.

Chau, F. (1999). Reticence and anxiety in language classrooms: with regard to F. 1 students in a Hong Kong secondary school, tesis de maestría dirigida por Mr. C. Barron, Universidad de Hong Kong (inédita), disponible en: http://hub.hku.hk/bitstream/10722/27586/1/FullText. pdf?accept=1, último acceso 1 de octubre de 2016.

Chen, T. (2003). "Reticence in class and on-line: two ESL students' experiences with communicative language teaching", en System, 31: 259-281.

Cheng, X. (2000). "Asians students'reticence revisited", en System, 28: 435-446.

Choe, Y. (1995). "Culture as a Background Knowledge in TEFL", en English Teaching, 50, 1: $141-156$.

Choi, J. Y. (2015). "Reasons for Silence: A Case Study of Two Korean Students at a US Graduate School", en TESOL Journal, 6, 3: 579-596.

Cortazzi, M. y Jin, L. (1996). "Cultures of learning: language classrooms in China", en H. Coleman (ed.), Society and the Language Classroom. Cambridge: Cambridge University Press, 169-206.

Donald, S. (2010). "Learning how to speak: Reticence in the ESL Classromm", en ARECLS: Annual Review of Education, Communication and Language Sciences, 7: 41-58.

Delima, E. (2012). "A Reticent Student in the Classroom: A Consequence of the Art of Questioning", en The Asian EFL Journal, 60: 50-69.

García Santa-Cecilia, A. (2009). "La enseñanza de ELE desde la perspectiva del currículo: logros y retos en la era de la globalización", en N. Arriaga et al. (eds.), El currículo de ELE en Asia-Pacifico. Selección de artículos del I Congreso de español como lengua extranjera en Asia-Pacifico. Manila: CE/LEAP, 17-38.

Guilloteaux, M. (2004). "Korean Teachers' Practical Understandings of CLT”, en English Teaching, 59, 3: 53-76.

He, X. (2008). "El silencio y la imagen china en la clase de ELE", Linred, 6: 1-20.

Hsieh, S. Y. (2015). "La Vulnerabilidad de la propia imagen y la Búsqueda de la perfección lingüística", Revista Nebrija de Lingüistica Aplicada a la Enseñanza de las Lenguas 19, disponible en: http://www.nebrija.com/revista-linguistica/la-vulnerabilidad-de-la-propiaimagen-y-la-busqueda-de-la-perfeccion-linguistica, última consulta: 1 de octubre de 2016. 
Jeon, J. (2009). "Key issues in applying the communicative approach in Korea: Follow up after 12 years of implementation", en English Teaching, 64, 4: 123-150.

Keaten, J. A. y Kelly, L. (2000). "Reticence: An Affirmation and Revision", en Communication Education, 49, 2: 165-177.

Keaten, J. A.; Kelly, L. y Finch, C. (1997). "Development of an instrument to measure reticence", en Communication Quarterly, 45, 1: 37-54.

Keaten, J. A.; Kelly, L. y Finch, C. (2000). "Effectiveness of the Penn State Program in Changing Beliefs Associated with Reticence", en Communication Education, 49, 2: 134-145.

Kelly, L.; Keaten, J. A.; Hazel, M. y Williams, J. A. (2010). "Effects of reticence, affect for communication channels, and self-perceived competence on usage of instant messaging", en Communication Research Reports, 27, 2: 131-142.

Kim, J. (2013). "Oral Communication Needs of Korean Students in a Business Communication Classroom", en Global Business Languages, 18: 81-96.

Kumaravadivelu, B. (2003). "Problematizing cultural stereotypes in TESOL", en TESOL Quarterly, 37, 4: 709-718.

Lee, G. (2009). "Speaking up: Six Korean students' oral participation in class discussions in US graduate seminars", en English for Specific Purposes, 28: 142-156.

Li, D. (1998). "It's Always More Difficult Than You Plan and Imagine": Teachers' Perceived Difficulties in Introducing the Communicative Approach in South Korea", en TESOL Quarterly, 32, 4: 677-703.

Li, X. y Jia, X. (2006). "Why Don't You Speak Up?: East Asian Students' Participation Patterns in American and Chinese ESL Classrooms", en Intercultural Communication Studies, 15, 1: $192-206$.

Littlewood, W. (1999). "Defining and Developing Autonomy in East Asian Contexts", en Applied Linguistics, 20, 1: 71-94.

Littlewood, W., (2000). "Do Asian students really want to listen and obey?", en ELT Journal, 54, 1: 31-34.

Liu, N. F. y Littlewood, W. (1997). "Why do many students appear reluctant to participate in classroom learning discourse?", en System, 25, 3: 331-384.

Liu, M. (2005a). "Causes of Reticence in EFL Classrooms: A Study of Chinese University Students", en Indonesian Journal of English Language Teaching, 1: 108-124.

Liu, M. (2005b). "Reticence in Oral English Language Classrooms: A Case Study in China", en TESL Reporter, 38, 1: 1-16.

Liu, M. y Jackson, J. (2008). "An exploration of Chinese EFL learners' unwillingness to communicate and foreign language anxiety", en Modern Language Journal, 92,1: 71-86.

Liu, M. y Jackson, J. (2009), "Reticence in Chinese EFL Students at Varied Proficiency Levels", TESL Canada Journal 26, 2: 65-81.

Liu, M.; Zhang, W. y Lu, Z. (2011). "Reticence and anxiety in Chinese university ESP poetry class: A case study, Journal of Language and Culture, 2, 2: 20-33.

Musytarif, K. (2015). Learners' reasons for being reticent in speaking class, trabajo final de grado en Enseñanza de inglés, dirigido por el Dr. Muhammad Amin, Universidad de Mataram (inédito).

O'Connell, M. (2010). "To text or not to text: Reticence and the utilization of short message services", en Human Communication, 13, 2: 87-102.

Phillips, G. M. (1965). "The problem of reticence", en Pennsylvania Speech Annual, 22: 22-38. Phillips, G. M. (1977). "Rhetoritherapy versus the medical model: Dealing with reticence", en Communication Education, 26: 34-43. 
Phillips, G. M. (1984). "Reticence: A perspective on social withdrawal", en J. A. Daly y J. C. McCroskey (eds.), Avoiding communication: Shyness, reticence and communication apprehension. Beverly Hills, CA: Sage, 51-66.

Phillips, G. M. (1986). "Rhetoritherapy: The principles of rethoric in training shy people in speech effectiveness", en W. H. Jones, J. M. Cheek y S. R. Briggs (eds.), Shyness: Perspectives on research and treatment. New York: Plenum Press, 357-374.

Phillips, G. M. (1991). Communication incompetencies: A theory of training oral performance behavior. Carbondale: Southern Illinois University Press.

Shimizu, J. (2006). "Why are Japanese Students Reluctant to Express Their Opinions In the Classroom?", en The Hiyoshi review of English studies, 48: 33-45.

Soo, R. y Goh, H. (2013). "Reticent Students in the ESL Classroom", en Advances in Language and Literary Studies, 4, 2: 65-73.

Swain, M. (1985). "Communicative competence: some roles of comprehensible input and comprehensible output in its development", en S. Gass y C. Madden (eds.), Input and Second Language Acquisition. Rowley, MA: Newbury House, 235-253.

Swain, M. y Lapkin, S. (1995). "Problems in output and the cognitive processes they generate: a step towards second language learning", en Applied Linguistics, 16, 3: 371-391.

Tran, T. (2013). "Is the learning approach of students from the Confucian heritage culture problematic?", en Educational Research for Policy and Practice, 12: 57-65.

Tsui, A. (1996), "Reticence and anxiety in second language learning", en K. Bailey y D. Nunan (eds.), Voices from the Language Classroom. Cambridge: Cambridge University Press, 145-167. 